\title{
FACTORS RELATING TO DEVELOPMENT OF CATHETER ASSOCIATED SEPSIS : A PROSPECTIVE STUDY
}

\author{
V. Ponnusamy ${ }^{1}$, V. Venkatesh ${ }^{2}$, A. Curley ${ }^{2}$, N. Brown ${ }^{3}$, C. Tremlett ${ }^{4}$, P. Clarke ${ }^{1}$ \\ ${ }^{I}$ Neonatal Intensive Care, Norfolk \& Norwich University Hospitals NHS Trust, Norwich, ${ }^{2}$ Neonatal Intensive \\ Care Unit, ${ }^{3}$ Clinical Microbiology, Cambridge University Hospitals NHS Foundation Trust, Cambridge, \\ ${ }^{4}$ Department of Microbiology, Norfolk \& Norwich University Hospitals NHS Trust, Norwich, UK
}

Background and aim: Insertion of percutaneous central venous lines (PCVLs) is an everyday procedure in the neonatal unit. The presence of PCVL is an important risk factor for catheter-related sepsis. We aimed to study the characteristics of infants with indwelling PCVLs who develop catheter-associated sepsis (CAS).

Design and methods: Prospective study in two tertiary neonatal units of all infants with a PCVL indwelling for $>24 \mathrm{~h}$. CAS was defined as an unwell baby with an indwelling PCVL who developed clinical signs of sepsis with no other cause for sepsis identified. We examined various factors/ characteristics to see which were associated with likelihood of development of CAS.

Results: 189 PCVLs were studied over a 14-month period. 47 neonates were clinically-septic at the time of line removal. Babies with clinically-suspected sepsis were significantly lower in gestational age and birth weight compared to well babies $(\mathrm{p}<0.01)$. Comparatively, PCVLs were inserted at an earlier postnatal age in well babies than in those with suspected sepsis $(p<0.01)$. In the suspected sepsis group, a significant proportion of the PCVLs were inserted via the lower limb $(\mathrm{P}=0.04)$ compared to well babies. Babies with suspected sepsis were significantly more likely to have had a smaller caliber PCVL inserted than well babies ( 28 gauge vs. 24 gauge, $\mathrm{p}=0.002$ ).

Conclusion: CAS occurs more commonly in babies of smaller gestation and birth weight. Preferential insertion of PCVLs via upper limbs may entail a lower risk of CAS because of the smaller length of in vivo foreign body liable to colonisation. 